\title{
Vitamin D deficiency is associated with increased risk of postural hypotension in older men: a cross-sectional analysis from The British Regional Heart Study
}

Artaza Gilani, Sheena Ramsay, Paul Welsh, Olia Papacosta, Lucy Lennon, Peter Whincup and S Goya Wannamethee

British Journal of General Practice

\section{Abstract}

Background There is growing interest in the role of vitamin D in extra-skeletal health, including postural hypotension. Postural hypotension is found in 1 in 5 community-dwelling adults aged 60 years and above. It increases risk of falls, fractures, cardiovascular disease and all-cause mortality. Better understanding of the aetiology of postural hypotension may help yield more effective treatment options than those that are currently available.

Aim The aim of this study was to investigate the association between circulating vitamin $D$, parathyroid hormone and postural hypotension.

Method This was a cross-sectional analysis of 3620 community-dwelling men living in the UK (mean age 68.6 years; standard deviation 5.5 years). Vitamin D status (nmol/L) was categorised as sufficient $(\geq 50)$, insufficient $(\geq 25-<50)$, or deficient $(<25)$. Parathyroid hormone levels were categorised by quintiles. Postural hypotension was defined by consensus criteria as a decrease in systolic blood pressure $\geq 20 \mathrm{mmHg}$ and/or diastolic blood pressure $\geq 10 \mathrm{mmHg}$ that occurred within three minutes of standing.

Results Compared to sufficient levels of vitamin $D$, vitamin $D$ deficiency increased risk of postural hypotension that specifically occurred within one minute of standing (OR $1.51,95 \% \mathrm{Cl}=1.06$ to 2.15) in multinomial, multiple logistic regression. Neither vitamin D insufficiency, nor elevated parathyroid hormone, were associated with postural hypotension.

Conclusion In this study, vitamin D deficiency was associated with increased risk of postural hypotension. Further research may help clarify whether treating vitamin $D$ deficiency can reduce the degree of postural hypotension, or if preventing the progression to vitamin $\mathrm{D}$ deficiency can reduce the incidence of postural hypotension. 